\title{
Sturm. Nie war mehr Hermeneutik in der Korpuslinguistik (gefragt)
}

\author{
Nicole M. Wilk
}

Eingegangen: 6. Mai 2021 / Angenommen: 20. Juli 2021 / Online publiziert: 3. November 2021

(C) Der/die Autor(en) 2021

Zusammenfassung Der Beitrag plädiert für eine höhere Sichtbarkeit und gezieltere Modellierung der qualitativ-hermeneutischen (Vor-)Entscheidungen, die in die Anwendung korpuslinguistischer Standardverfahren eingelassen sind. Anhand verschiedener Fallbeispiele wird die datengeleitete, nicht intentionalistische Zusammenstellung von Belegsammlungen als Vorzug quantitativer Verfahren erkannt. Die Verbindung von Kollokationsnetzen mit Deutungsmustern, von Wortverbindungen mit Verstehenshorizonten, an deren Schnittstellen zentrale sprachliche Einheiten als Knotenpunkte liegen, wird mithilfe verschiedener korpus- und soziolinguistischer Ansätze modelliert. In der Frage, wie korpushermeneutisch das Herausbilden verschiedener Lesarten im Diskurs (Diskurssalienzen) begründet und erfasst werden kann, liegt das Hauptaugenmerk auf dem Prozess der Ko-Konstruktion zwischen Algorithmus und menschlicher Interpretationsinstanz als eine Art Anti-Hermeneutik ohne vorgängigen Code oder Symbolismus.

Schlüsselwörter Methodentriangulation · Kollokationsnetz · Anti-Hermeneutik · Diskurssalienz 


\title{
Storm. Never Has There Been More Hermeneutics (in Demand) in Corpus Linguistics
}

\begin{abstract}
The paper argues for a higher visibility and more targeted modelling of the qualitative-hermeneutic (pre-)decisions embedded in the application of standard corpus linguistic procedures. On the basis of various case studies, the non-intentionalist data driven handling of utterance collections is recognised as an advantage of quantitative corpus linguistic. The connection of collocation networks with patterns of interpretation, of word connections with horizons of understanding (at the intersections of which lie central units of meaning as nodes) is modelled with the help of different corpus and sociolinguistic approaches. In the question of how the emergence of different readings in discourse (discourse salience) can be justified and captured corpus-hermeneutically, the main focus is on the process of co-construction between algorithm and human interpreter as a kind of anti-hermeneutics without any antecedent code or symbolism.
\end{abstract}

Keywords Method Triangulation · Collocation Net · Anti-Hermeneutics · Discourse Salience

Der Wetterbericht hat ein hohes Potenzial für die Korpuslinguistik: Er kann mit seinen durchziehenden Hoch- und Tiefdruckgebieten und den vereinzelten Schauern gattungstypische Sprachgebrauchsmuster veranschaulichen (vgl. Bubenhofer 2009, S. $44 \mathrm{f}$.), er kann phraseologisch die paradigmatischen Beziehungen zwischen Wörtern (Sturm) und Syntagmen (frische Brise) belegen (vgl. Burger 2010, S. 55) und er führt sehr schnell zu den Fallstricken der digitalen Textanalyse, wovon ich im Folgenden zwei skizzieren möchte. Es geht erstens um die Frage einer funktionalen Äquivalenz von Bestimmungswörtern und Attributen. Verbindungen mit dem Wetterlexem Sturm können dies veranschaulichen: Sturmböen und stürmische Böen sind in der Fachsprache der Meteorologie nicht dasselbe, denn Sturmböen haben per definitionem eine höhere Geschwindigkeit als stürmische Böen. Zweitens handelt es sich - und dies lässt sich ebenfalls am Beispiel der Verwendungsweisen von NN Sturm zeigen - um den altbekannten Fall, dass unterschiedliche Lesarten ein und derselben Ausdrucksgestalt (wie Sturm) korpuslinguistisch zwar erfasst, aber nur schwer systematisch für alle Korpora, Zeiten etc. erhoben werden können. Hinzu kommen phraseologische Besonderheiten: Der Sturm im Wasserglas ist semantisch durch seine hohe Idiomatizität ein völlig anderer als der Sturm auf hoher See etc. Beide Fallstricke verdeutlichen den (notwendigen) Beitrag hermeneutischer Prozesse in der quantitativen Korpusanalyse, der durch den Materialismus der Form oft nicht explizit verhandelt wird, der aber gerade durch diesen in besonderer Weise herausgefordert ist. Die Formgestalt wird in der Korpuslinguistik des britischen Kontextualismus konsequent zum Angelpunkt der semantischen Beschreibung von 
Variation: Alles ausdrucksseitig Verschiedene ist semantisch getrennt ${ }^{1}$ und bildet eigene Kollokationsnetzwerke aus. Das Sinclair'sche Motto »trust the text« vollzieht sich in diesem Sinne als Vernetztheitsanalyse für einzelne Wortformen, so dass die unterschiedlichen Bedeutungen durch Verschiebungen und Kontraste in den jeweiligen kollokativen Netzwerken fassbar werden. Für Sinclair (2004, S. 19) verbindet sich $»$ the coincidence of distinct environmental patterns with the shades of meaning of a word.«

Die semantische Prägung durch die »environmental patterns « fällt nun bezogen auf das Sturm-Beispiel für die NP stürmische Böen primär wettersprachlich aus, während jedoch die meteorologische Gebrauchsweise von NN Sturmböen nur eine unter vielen ist: Korpuslinguistisch erreicht das Plural-NN Sturmböen nicht nur semantisch höhere Geschwindigkeiten als die NP stürmischen Böen, es hat auch im DeReKo eine zehnmal höhere Frequenz gegenüber der Suchanfrage stürmische* Böen. Und doch sind beide durch ihre Gebrauchsnorm für den Wetterbericht kontextualisierend. Beim Vergleich ihrer Kookkurrenzprofile stößt man auf viele identische Kollokatoren wie Gewitter, Regengüsse, Starkregen und Hagel, die typischerweise in der Textsorte Wetterbericht vorkommen. Auch ist eher nicht zu vermuten, dass das NN Sturmböen im Wetterbericht zehnmal häufiger auftritt als die NP stürmische Böen, etwa weil Sturmböen berichtenswerter sind oder als Wetterphänomen so viel häufiger auftreten. Die Durchsicht der Kookkurrenzen ergibt recht schnell, dass das NN Sturmböen noch eine weitere Gebrauchsdomäne besitzt als die der Deskription einer Windstärke. Darauf deutet der auf Rang 7 platzierte erste Adjektivkollokator heftig hin. Die Belege für die Kollokation heftige Sturmböen stammen jedoch nur selten aus einem Wetterbericht. Während also im Wetterbericht vermutlich paritätisch von Sturmböen und stürmischen Böen die Rede ist, besitzt das Kompositum Sturmböen noch mindestens ein weiteres Handlungsfeld, das frequenzbezogen gegenüber dem ersten dominiert und verstärkt im Bereich der Pressekommunikation auftritt. Diese journalistische Lesart wird von etlichen syntagmatischen Mustern zu Kollokatoren des NN Sturmböen indiziert wie Bäume entwurzelten/knickten, Gefahr besteht/steigt sowie erhebliche Schäden angerichtet. Syntaktisch ist das NN dabei häufig agentiviert und gewinnt den Charakter einer Verursachung, wenn z.B. Sturmböen Bäume entwurzeln und dabei als agentivierte Protagonisten Chaos oder Schäden anrichten. Sturmböen sind teils noch agenshafter modelliert und werden zu metaphorischen Aggressoren, wenn sie über den Berg toben, einen Ort heimsuchen, über den Boden peitschen und Dächer abdecken. Diese journalistische Variante des NN Sturmböen als einer für den Menschen gefährlichen und handlungsmächtigen Naturerscheinung tritt im Wetterbericht nicht in Erscheinung, denn sie wird dort kontextuell nicht lizenziert. Oder vielleicht doch? Sind nicht Lexeme durch ihre Ausdrucksgestalt diachron und transdiskursiv vernetzt? Denken wir nicht auch dann, wenn wir Nomen wie Sturm, Orkan, Taifun, Tsunami im Wetterbericht hören, an Si-

\footnotetext{
${ }^{1}$ Damit verbunden ist die Entdeckung, dass selbst flexionsmorphologische Variation z. B. durch Numerus (Singular/Plural) für den Einbettungscharakter der jeweiligen Wortform in Diskurse erhebliche Auswirkung haben kann, d.h. sie können in unterschiedliche Sprachgebrauchsmuster eintreten. So verbindet sich bspw. im Englischen das NN year eher mit Ordinalzahlen (first, second ... year), während die Pluralform years eher in festen Wortverbindungen wie a couple of years vorkommt, die ganz andere Kontexte evozieren (vgl. Sinclair/Jones/Daley 2004, S. 119 f.).
} 
tuationen der in journalistischen Texten berichteten und entworfenen Katastrophen oder Ausnahmezustände?

Für Wortformen wie das Plural-NN Sturmböen lassen sich verschiedene Verstehenshorizonte anhand der Kollokationsprofile beschreiben. Das Sturmböen-Beispiel aus dem Wetterbericht wirft die Frage nach der mehrdimensionalen Semantik von Wortformen auf, die in verschiedenen Diskursen zirkulieren und dort unterschiedliche Muster ausbilden. In diesem Sinne möchte ich mit Bezug auf Sinclair dafür plädieren, das korpuslinguistische Credo in eine hermeneutische Richtung zu erweitern (was in der Praxis der Mehrheit der Korpusanalysen bereits geschieht) hin $\mathrm{zu}>$ trust the text and single out the variants $<$. Die Grundannahme hierbei ist, dass sprachstatistische Werte (Kookkurrenzen, über Kontrastkorpora ermittelte signifikante Schlüsselwörter etc.) auf verschiedene Weise stratifizierbar sind und je nach Fragestellung diskurslinguistisch unter anderen Gesichtspunkten, d.h. z.B. Deutungsmustern, diskurssemantischen Grundfiguren, Themen, Gattungen oder Topoi sortiert werden können. Durch diskurssemantische Verfahren tritt die Diskurslinguistik das Erbe hermeneutischer Ansätze an (vgl. Bär 2016, S. 282), und es ist m.E. Aufgabe einer korpuslinguistisch fundierten Diskursanalyse, weitere Filterprozesse in Form von soziopragmatisch motivierten Suchanfragen zu initiieren. Das Reduzieren und Zuordnen von Varianten vor dem Hintergrund einer sozio-, diskursoder kulturlinguistisch motivierten Fragestellung ist im Kern ein hermeneutischer Prozess. Mit Verweis auf den zirkulierenden Charakter korpuslinguistischer Methoden bezeichnen Dang-Anh und Rüdiger diesen Prozess als korpushermeneutisch: »[W]e reassess the quantitative analytical steps by corpus-hermeneutic reflections « (Dang-Anh/Rüdiger 2015, S. 58). Der Output dieser Reorganisation ist eine Belegauswahl, die Konstellationen z. B. als »historische Sinnwelten« (Schuster 2019, S. 224) oder als Markierung sozialer Ordnungsmuster erfasst. Aber hängt dieses Auswählen von Varianten mit Textverstehen im Sinne der hermeneutischen Interpretation zusammen? Auch wenn der Terminus >Hermeneutik $<$ in den Arbeitsfeldern der germanistischen Linguistik eher ungebräuchlich geworden zu sein scheint und der Stellenwert der Hermeneutik für die Linguistik kontrovers diskutiert wird, kommen doch interpretative Verfahren gerade in den Bereichen wieder vermehrt zum Einsatz, in denen sich die Linguistik derzeit am rasantesten entwickelt und zwar im quantifizierenden korpuslinguistischen Umgang mit großen Datenmengen. Gerade hier werden hermeneutische Vorgehensweisen mitunter als konkurrierende Methoden wahrgenommen, obwohl sie an corpus-driven-Studien im Hintergrund maßgeblich beteiligt sind.

Hingegen wurden in anderen sprach- und konversationsanalytischen Ansätzen interpretative Verfahren vielfach in Anpassung an einen erweiterten Analyserahmen (weiter-)entwickelt. Die Deutung wird dabei nicht dem:r einzelnen Analysierenden überlassen, sondern aus dem multimodalen Zusammenspiel oder aus den interaktiven Relevanzen abgeleitet. Bspw. dient das Transkript in der Gesprächsanalyse der Sichtbarkeit verschiedener im Gespräch zusammenwirkender Modalitäten und sichert Interpretationen über die ratifizierenden Signale aller Gesprächsbeteiligten ab: Gelästert wurde, wenn jemand in die Lästernarration eingestiegen ist, Streit ereignet sich dann, wenn eine Äußerung oder Geste nachweislich als Provokation aufgefasst wurde, eine Frage vollzieht sich erst durch die konditionell relevant gesetzte Ant- 
wort. Auch die Raum- und Interaktionslinguistik hat ihren Untersuchungsgegenstand durch die Analyse derjenigen Faktoren ausgeweitet, die im Umfeld in spezifischer Weise zur Herstellung eines Deutungsmusters beitragen.

In der digitalen Linguistik ist womöglich der Beitrag hermeneutischer Prozesse dadurch verdeckt, dass der Schritt der Datenbeschreibung nicht durch dieselbe interpretierende Person ausgeführt wird, die auch die Fragestellung verfolgt, sondern durch einen Algorithmus. Doch die Korpusanalyse gewinnt beim Korpuszuschnitt bereits hermeneutische Züge, noch bevor der Computer überhaupt zum Einsatz kommt. Die Zusammenstellung der Analysedokumente lenkt nicht nur den Blick auf den Gegenstand, sie konstituiert diesen auch:

»(...) [I]t is important to understand that the dataset itself can be an important determining factor in shaping, for example, the social categories used within sociolinguistic/pragmatic annotation schemes - and, in turn, our interrogations of the dataset« (Archer 2018, S. 332).

Eine hermeneutisch fundierte Sprachwissenschaft untersucht nach Hermanns (2012, S. 68) »wie Sprache funktioniert« und dies ist auch Ziel einer digitalen Korpuslinguistik. Auch sie interessiert wie jede andere linguistische Teildisziplin »das Zusammenspiel von Zu-Verstehen-Geben und Verstehen« (Hermanns 2012, S. 69), nur dass im Falle der Computerauswertung die Einheiten, die potenziell etwas zu verstehen geben, mithilfe mathematischer Verfahren ermittelt wurden. »Eine Linguistik ohne Hermeneutik ist ein Unding «, heißt es bei Hermanns (2012, S. 69) weiter und dies nimmt weder die Korpuspragmatik noch die digitale Linguistik aus. Auch wer Algorithmen einsetzt, interessiert sich dafür, wie durch Ausdrucksformen Sinn hergestellt wird.

Doch welche Art von Hermeneutik ist in die quantitative Diskursanalyse eingelassen? Können seriell ermittelte Konstruktionsmuster mit Ausdruckseinheiten verglichen werden, die durch kriteriengestützte empathische Lektüren auffällig geworden sind? Ist das Hochfrequente immer auch das (diskursiv) Entscheidende, wenn doch wie im Beispiel von Sturmböen die wetterlexikalische Einbindung nur auf ein Zehntel aller Fälle zutrifft? Das hermeneutische Vorgehen präsentiert sich in der Korpusanalyse als Rahmungsversuch mit abduktivem Charakter nach dem Muster >Wenn diese oder jene Deutung zuträfe, dann könnten die Kollokatoren verschiedenen Mustern zugeordnet werden und die heterogene Ergebnisliste würde nicht weiter verwundern $<$.

Die Belegsammlung ist somit Grundlage eines hermeneutischen Interpretierens, insofern sie Belege von etwas enthält, das nach grammatischen, soziopragmatischen o. a. Gesichtspunkten an semantische Informationen und Rahmungen rückgebunden werden kann. Dieser hermeneutische Zug korpuslinguistischen Arbeitens wird u.a. unter dem Stichwort Methodentriangulation intensiv diskutiert. Da das Erfassen von Varianten und die Verteilung unterschiedlicher Korpuszuschnitte Korpusanalysen auf verschiedenen Ebenen erfordert, besteht für Egbert und Baker (2016, S. 204) die zentrale Herausforderung für die Triangulation darin, die Expertise von mehreren Forschenden zu verbinden und damit auch die Qualität der Ergebnisse zu erhöhen: 
»Another benefit of collaboration among researchers is the possibility of combining two or more different skill sets to the same data. We believe this is the ideal scenario for effectively applying methodological triangulation to a corpus. Not only does this save researchers from having to become experts in a novel research methodology, it also offers greater credibility and quality to the study.«

Ein Vorzug der algorithmenbasierten Auswertung sprachlicher Daten auch für das inhärente hermeneutische Vorgehen wird jedoch selten genannt: Es handelt sich bei algorithmengestützten Analysen um ein konsequent anti-intentionalistisches Vorgehen. In diesem Sinne der Textoberfläche zu vertrauen, gibt jegliche Versuche preis, im Text angelegte Bedeutungsweisen rekonstruieren zu wollen. Diskurs ist, in diesem methodischen Zugang im Foucault'schen Sinn einer Vielheit von Positivitäten, Ergebnis dessen, was diskutiert, aufgenommen, weitergedacht wurde und nicht das, was Autor:innen vermeintlich »wollen«. Algorithmen erfassen Musterbildung im Rücken der Subjekte zudem unabhängig von einem wie auch immer ausgeprägten sozialen Symbolismus. Der Computer kennt keine gesellschaftlichen Codes und fokussiert Frequenz und Vergleich, gelenkt durch die forschenden Personen, die bestimmen, in welchen Textsammlungen Frequenzen erhoben und welche Einheiten kombiniert und verglichen werden. Diese Auswahlprozedur erinnert an das, was Laplanche etwas provokativ eine Anti-Hermeneutik genannt hat (Laplanche 1998). Er bezeichnet damit die psychoanalytische »Übersetzung «, die zwar auch eine Art der Deutung ist (Freud selbst spricht von Traumdeutung), die aber ohne vorgegebenen Symbolismus operiert. Sie stößt irgendwann (ursprünglich im Patientengespräch) eher zufällig auf symbolische Einheiten und interpretiert diese probeweise. In Laplanches anti-hermeneutischer Auslegung der psychoanalytischen Methode enthält der Text (z. B. die Traumerzählung) eine Botschaft des anderen und diese Botschaft des anderen zu übersetzen, bedeutet für Freud in Laplanches Verständnis, auf ihn zu reagieren, d.h. nicht zu deuten, sondern zu übersetzen, in welcher Weise sich der andere an jemanden, der interpretiert, wendet. Diese interaktive Bedeutungskonstitution wird in der Linguistik für das Verstehen im Interaktionszusammenhang als Ko-Konstruktion ausgewiesen (vgl. Dausendschön-Gay/Gülich/Krafft 2015): Deutungen, Situationen, Gesprächsgattungen, Machtkonstellationen etc. sind somit ein »Gemeinschaftsprodukt « der alltäglichen Interaktion. Und insofern Sinnhaftes nur aus dem entsteht, was bestätigt und fortgeführt wird, sind Auswahl und Annotation der Varianten Übersetzungen der »Botschaften« des Computers (oder der Sprache selbst?) seitens der interpretierenden Instanz in einen soziokulturell motivierten Interpretationsrahmen. Die damit behauptete Ko-Konstruktion zwischen Algorithmus und menschlicher Interpretationsinstanz beginnt streng genommen nicht erst mit der Sichtung der ausgegebenen Wortfrequenzen, Kollokationen, Worteinbettungen etc. »Auch die Software prägt die semantische Interpretation der Ausdrücke « (Haß 2007, S. 243) und vielleicht ließe sich ebenfalls im Sinne der Ko-Konstruktion ergänzen, dass es die Anwendungs- und Verständnisweisen der Software sind, die Deutungen hervorbringen, also all die manchmal schwer explizierbaren forschungspraktischen Begründungen für ein Signifikanzmaß, ein Kontrastkorpus, ein Exportformat oder eine Visualisierung, denn schlussendlich stützt auch jede Wahl der Darstellungsform die präferierten Interpretationen (vgl. Bubenhofer 2020). 
Das Zusammenspiel aus quantitativen Analysen und Auswahl ist rekursiv und potenziell unbegrenzt. Teilweise sind Ansätze der Diskurs- und Interaktionslinguistik bereits konzeptionell in die korpusanalytischen Standardberechnungen integriert. So laufen bspw. beim Konzept des Schlüsselworts (vgl. Stubbs 2008) Interpretationslinien zusammen, die den Schlüsselcharakter einer Phrase u. a. anhand von Grundfragen für soziale Gruppen fundieren (vgl. Liebert 2003). Ebenso werden Wortfrequenzen und Signifikanzen in Bezug zu zeitlichen Verteilungen, Gattungen, Diskursen etc. gesetzt.

Der Terminus der Salienz mag ganz gut geeignet sein, um diese Vermitteltheit zwischen statistischer Evidenz und kultureller Deutung einzufangen (vgl. Pfänder et al. 2013, S. 22). Jedoch müsste hier methodologisch eine Brücke zwischen den diskurssteuernden salienten Ausdruckseinheiten - z. B. ein Schlüsselzitat im politischen Diskurs (vgl. Klein 2017) - und der korpuslinguistischen Erfassung eines Kollokationsnetzwerks geschlagen werden. Im Sinne des korpuslinguistischen Anspruchs, corpus driven sprachliche Einheiten hinsichtlich einer konkreten Fragestellung aufzuspüren, sind der »eigentliche Gegenstand der Analyse linguistischer Korpora (...) weder Wörter noch Texte, sondern das, was Diskurse als intertextuelle Zusammenhänge aus Wörtern machen.«(Haß 2007, S. 259f.) Je mehr diese Art der Diskursanalyse verstanden als Kollokationsanalyse voranschreitet, umso blasser werden jene sprachlichen Einheiten, die als Knoten- oder Ausgangspunkt für die Beschreibung der intertextuellen Zusammenhänge genommen wurden.

In welchen intertextuellen Zusammenhängen hat sich nun der Sturm aus dem Wetter- und Nachrichtendiskurs verfangen? Als ein für den Menschen mitunter lebensgefährliches Naturphänomen mit hohem Nachrichtenwert war der Sturm auch schon historisch »attraktiv«, um Gefährlichkeit und Angriffsstärke zu signalisieren. Das NN Sturm stiftet also in ganz unterschiedlichen Epochen Diskurssalienzen. In den Korpora der NS-Zeit ${ }^{2}$ findet es sich eingebunden in das rekurrente Syntagma Dem Sturm wird die Berechtigung verliehen (...) künftig die Bezeichnung »Sturm ZAHL NAME«zu führen. Der Sturm war im Nationalsozialismus eine Einheit der sog. Sturmabteilung (SA), die drei Trupps umfasste. Drei bis zehn Stürme wurden zu einem Sturmbann zusammengefasst (https://de.wikipedia.org/wiki/Sturmabteilung). Die Sturmabteilung schreckte nicht vor Gewalt zurück. Sie verhalf Hitler zur Macht, indem sie Kritiker angriff und ermordete, wobei »hinsichtlich der Gewalt gegen Außenstehende die Grenzen zwischen dem Politischen und dem Privaten verschwammen « (Hein 2012, S. 61).

Gegenwartssprachlich springen auch unpolitische, eher spielerische Kontexte ins Auge, die nicht mit physischer Gewalt zusammenhängen. So ist das Sturmteam oder der Stürmer im Fußball zwar angriffslustig, aber effektiv nur im Hinblick auf das Ziel des Toreschießens. Gibt man den Ausdruck Sturm in eine der gängigen Suchmaschinen ein, führt dies in erster Linie zum Titel einer Vorabendserie voller romantischer Klischees über den »Sturm der Liebe«. All diese Gebrauchsvarianten sind durch den

\footnotetext{
2 Die NS-Korpora sind im Rahmen des DFG-Projekts zur >Sprachlichen Sozialgeschichte 1933-1945< unter der Leitung von Heidrun D. Kämper (IDS Mannheim) und an der Universität Paderborn im DFGProjekt >Heterogene Widerstandskulturen - Sprachliche Praktiken des Sich-Widersetzens von 1933-1945< unter der Leitung von Britt-Marie Schuster entstanden und projektintern über Cosmas verfügbar.
} 
inhärenten Aspekt der unkontrollierbaren Naturgewalt metaphorisiert. Das Moment des drohenden Kontrollverlusts hat jedoch in den verschiedenen Diskursen nicht nur völlig unterschiedliche Valenzen, inhaltlich-thematische Kontexte etc., es ist wohl auch weder sinnvoll noch einleuchtend, den Sturm in Sturm der Liebe mit dem in Sturm der Liga und dem Sturm in Sturm der Sturmabteilung mit dem Sturm des Wetterberichts diskurssemantisch in Verbindung zu bringen. Selbst der kollokativ in ganz unterschiedlichen Diskursen verfestigte Sturm der Empörung oder Entrüstung hat mit dem Sturm der Liebe diskursanalytisch nicht mehr gemeinsam, als dass beide in derselben Rankingtabelle nebeneinander aufgeführt sind. Zudem enthält die NP Sturm der Liebe im DeReKo mit ihren rekurrenten Anführungszeichen fast ausschließlich Referenzen auf den Titel der Telenovela. Die Verschiedenheit der Kontextualisierungsprofile erfordert eine neue Ordnung z. B. nach Handlungsund Wissensfeldern oder nach Aspekten der Wirklichkeitskonstitution. Diese Sortierung kann die Korpuslinguistik keinesfalls ohne den »Sturm« der Hermeneutik bewältigen, verstanden als dasjenige, was jenseits geplanter und standardisierter Verfahren Schneisen in Belegsammlungen schlägt - auf die »Gefahr « hin, dass die eine Ordnung plausibler, gesellschaftskritischer, anschlussfähiger etc. ist als die andere. Dabei geht es nicht primär um die Explikation von Lesarten, sondern mehr um Fragen nach den sprachlichen Bedingungen und Faktoren für das Prozessieren von Diskursen: Wie bildet ein Ausdruck Bedeutung im Diskurs heraus, welche Funktion hat er, was stellt er her, was setzt er durch, was reguliert er, wogegen ist er gerichtet?

Erst durch die soziokulturelle Strukturierung von Korpusdaten können Gebrauchsweisen in Abgrenzung zu alternativen Themenfeldern, Akteursgruppen etc. beurteilt werden, so dass auch niederfrequente Vorkommen in bestimmten Kontexten Salienz gewinnen. Bspw. handelt es sich bei der Verwendung des NN Sturm in einem Artikel aus dem deutschen Widerstand um eine Ausnahmeerscheinung. In dem gegen den Hitlerfaschismus gerichteten kommunistischen Informationsund Kampforgan Der Vorbote von 1941 ist das NN Sturm etwas abseits vom Vers platziert, eingefasst in Gedankenstriche wirkt das Wort wie ein Aufruf, der vor dem Hintergrund der Sturm-Verwendungen in der NS-Propaganda zu einem Gegensturm anhebt.

An die Kleinmütigen

Was fliehst du eilend vor der Welt,

Sie bleibt dir doch zur Seite!

Drum sei ein Mann - und sei ein Held!

Und stell' dich ihr zum Streite. — Sturm -

(zitiert nach Oppenheimer 1969, S. 192)

Der Aufruf zum Sturm steht aufgrund einer Reihe von Merkmalen - nicht zuletzt durch die Verbindung mit dem NN Streite und dem Appellcharakter der Zeilen - ganz im Zeichen des Widerstands. Doch er kann in seinem diskursiven Widerspruchssinn nur unter der Bedingung soziopragmatisch strukturierter, d. h. interpretierend gesichteter Korpusdaten erschlossen werden. 
Funding Open Access funding enabled and organized by Projekt DEAL.

Open Access Dieser Artikel wird unter der Creative Commons Namensnennung 4.0 International Lizenz veröffentlicht, welche die Nutzung, Vervielfältigung, Bearbeitung, Verbreitung und Wiedergabe in jeglichem Medium und Format erlaubt, sofern Sie den/die ursprünglichen Autor(en) und die Quelle ordnungsgemäß nennen, einen Link zur Creative Commons Lizenz beifügen und angeben, ob Änderungen vorgenommen wurden.

Die in diesem Artikel enthaltenen Bilder und sonstiges Drittmaterial unterliegen ebenfalls der genannten Creative Commons Lizenz, sofern sich aus der Abbildungslegende nichts anderes ergibt. Sofern das betreffende Material nicht unter der genannten Creative Commons Lizenz steht und die betreffende Handlung nicht nach gesetzlichen Vorschriften erlaubt ist, ist für die oben aufgeführten Weiterverwendungen des Materials die Einwilligung des jeweiligen Rechteinhabers einzuholen.

Weitere Details zur Lizenz entnehmen Sie bitte der Lizenzinformation auf http://creativecommons.org/ licenses/by/4.0/deed.de.

\section{Literatur}

Archer, Dawn (2018): Context and historical (socio)pragmatics twenty years on. In: Journal of Historical Pragmatics 18/2, S. 315-336.

Bär, Jochen (2016): Text- und Diskurshermeneutik. In: Muttersprache 4, S. 281-301.

Bubenhofer, Noah (2009): Sprachgebrauchsmuster. Korpuslinguistik als Methode der Diskurs- und Kulturanalyse. Berlin/New York: de Gruyter.

Bubenhofer, Noah (2020): Visuelle Linguistik. Zur Genese, Funktion und Kategorisierung von Diagrammen in der Sprachwissenschaft. Berlin: de Gruyter.

Burger, Harald ( $\left.{ }^{4} 2010\right)$ : Phraseologie. Eine Einführung am Beispiel des Deutschen. Berlin: Erich Schmidt.

Dang-Anh, Mark/Rüdiger, Jan Oliver (2015): From frequency to sequence: How quantitative methods can inform qualitative analysis of digital media discourse. In: 10plus1: Living Linguistics 1, S. 57-73.

Dausendschön-Gay, Ulrich/Gülich, Elisabeth/Krafft, Ulrich (Hg.) (2015): Ko-Konstruktionen in der Interaktion. Die gemeinsame Arbeit an Äußerungen und anderen sozialen Ereignissen. Bielefeld: transcript.

Egbert, Jesse/Baker, Paul (2016): Research Synthesis. In: Dies. (Hg.): Triangulating Methodological Approaches in Corpus-Linguistic Research. New York/London: Routledge, S. 183-208.

Haß, Ulrike (2007): Korpus-Hermeneutik. Zur hermeneutischen Methodik in der lexikalischen Semantik. In: Fritz Hermanns/Werner Holly (Hg.): Linguistische Hermeneutik. Theorie und Praxis des Verstehens und Interpretierens. Tübingen: Niemeyer, S. 241-261.

Hein, Bastian (2012): Elite für Volk und Führer? Die Allgemeine SS und ihre Mitglieder 1925-1945. Quellen und Darstellungen zur Zeitgeschichte. Herausgegeben vom Institut für Zeitgeschichte. München: Oldenbourg.

Hermanns, Fritz (2012): Sprachgeschichte als Mentalitätsgeschichte. Überlegungen zu Sinn und Form und Gegenstand historischer Semantik. In: Heidrun Kämper/Angelika Linke/Martin Wengeler (Hg.): Der Sitz der Sprache im Leben. Beiträge zu einer kulturanalytischen Linguistik. Berlin/Boston: de Gruyter.

Klein, Josef (2017): Saliente Sätze. In: Kersten Sven Roth/Martin Wengeler/Alexander Ziem (Hg.): Handbuch Sprache in Politik und Gesellschaft. Berlin/Boston: de Gruyter, S. 139-164.

Laplanche, Jean (1998): Die Psychoanalyse als Anti-Hermeneutik. In: Psyche 52, S. 605-618.

Liebert, Wolf (2003): Zu einem dynamischen Konzept von Schlüsselwörtern. In: Zeitschrift für Angewandte Linguistik 38, S. 57-83.

Oppenheimer, Max (1969): Der Fall Vorbote. Zeugnisse des Mannheimer Widerstandes. Frankfurt a. M.: Röderberg.

Pfänder, Stefan/Behrens, Heike/Auer, Peter/Jacob, Daniel/Kailuweit, Rolf/Konieczny, Lars/Kortmann, Bernd/Mair, Christian/Strube, Gerhard (2013): Erfahrung zählt. Frequenzeffekte in der Sprache - ein Werkstattbericht. In: LiLi - Zeitschrift für Literaturwissenschaft und Linguistik 43/169, S. 7-32.

Schuster, Britt-Marie (2019): Sprachgeschichte als Geschichte von Texten. In: Jochen Bär/Anja Lobenstein-Reichmann/Jörg Riecke (Hg.): Handbuch Sprache in der Geschichte. Berlin/Boston: de Gruyter, S. 219-240.

Sinclair, John (2004): Trust the Text: Language, Corpus and Discourse. London/New York: Routledge. 
Sinclair, John/Jones, Susan/Daley, Robert (2004): English Collocation Studies. The OSTI Report. London/New York: Continuum International Publish Group.

Stubbs, Michael (2008): Three Concepts of Keywords. In: Marina Bondi/Mike Scott (Hg): Keyness in Texts. Amsterdam/Philadelphia: John Benjamins, S. 21-42. 